\title{
Understanding of Colistin Usage in Food Animals and Available Detection Techniques: A Review
}

\author{
Harsh Kumar ${ }^{1}$, Bing-Huei Chen ${ }^{2}$, Kamil Kuca ${ }^{3,4, * \mathbb{D}}$, Eugenie Nepovimova ${ }^{3}$, Ankur Kaushal ${ }^{5}$, \\ Rupak Nagraik ${ }^{1}$, Shashi Kant Bhatia ${ }^{6}{ }^{\circledR}$, Daljeet Singh Dhanjal ${ }^{7}{ }^{\mathbb{D}}$, Vinod Kumar ${ }^{8}$, \\ Anil Kumar ${ }^{1}\left(\mathbb{D}\right.$, Navneet Kumar Upadhyay ${ }^{9}\left(\mathbb{C}\right.$, Rachna Verma ${ }^{10}{ }^{(1)}$ and Dinesh Kumar ${ }^{1, *(1)}$ \\ 1 School of Bioengineering \& Food Technology, Shoolini University of Biotechnology and Management \\ Sciences, Solan 173229, H.P., India; microharshs@gmail.com (H.K.); rupak.nagraik@gmail.com (R.N.); \\ kumaranil@shooliniuniversity.com (A.K.) \\ 2 Department of Food Science, Fu Jen Catholic University, New Taipei City 24205, Taiwan; \\ 002622@mail.fju.edu.tw \\ 3 Department of Chemistry, Faculty of Science, University of Hradec Kralove, 50003 Hradec Kralove, \\ Czech Republic; eugenie.nepovimova@uhk.cz \\ 4 Biomedical Research Center, University Hospital Hradec Kralove, 50003 Hradec Kralove, Czech Republic \\ 5 Centre of Nanotechnology, Amity University, Manesar, Gurugram-122413, Haryana, India; \\ ankur.biotech85@gmail.com \\ 6 Department of Biological Engineering, College of Engineering, Konkuk University, Seoul 05029, Korea; \\ shashikonkukuni@konkuk.ac.kr \\ 7 School of Bioengineering and Biosciences, Lovely Professional University, Phagwara 144411, Punjab, India; \\ daljeetdhanja192@gmail.com \\ 8 School of Water, Energy and Environment, Cranfield University, Cranfield MK430AL, UK; \\ Vinod.Kumar@cranfield.ac.uk \\ 9 School of Pharmaceutical Sciences, Shoolini University of Biotechnology and Management Sciences, \\ Solan 173229, H.P., India; navneetqa@gmail.com \\ 10 School of Biological and Environmental Sciences, Shoolini University of Biotechnology and Management \\ Sciences, Solan 173229, H.P., India; rachnaverma@shooliniuniversity.com \\ * Correspondence: kamil.kuca@uhk.cz (K.K.); dineshkumar@shooliniuniversity.com(D.K.); \\ Tel.: +420-603-289-166 (K.K.)
}

Received: 14 September 2020; Accepted: 8 October 2020; Published: 16 October 2020

check for updates

Simple Summary: Colistin is a last resort drug for the treatment of infection caused by multidrug-resistant Gram-negative bacteria. Different studies have uncovered the negative impact of colistin consumption in animals. Therefore, it has become essential to monitor the dosing regimens of colistin and assess their negative effects. The current review intends to provide brief information of colistin usage and its associated negative impact and discuss available techniques to detect colistin in animal-based food so that effective preventive measures can be taken to minimize the health risks in both animals and humans.

Abstract: Progress in the medical profession is determined by the achievements and effectiveness of
new antibiotics in the treatment of microbial infections. However, the development of multiple-drug
resistance in numerous bacteria, especially Gram-negative bacteria, has limited the treatment options.
Due to this resistance, the resurgence of cyclic polypeptide drugs like colistin remains the only option.
The drug, colistin, is a well-known growth inhibitor of Gram-negative bacteria like Acinetobacter
baumanni, Enterobacter cloacae, Klebsiella pneumoniae, and Pseudomonas aeruginosa. Technological
advancements have uncovered the role of the mcr-1(mobilized colistin resistance) gene, which is
responsible for the development of resistance in Gram-negative bacteria, which make them distinct
from other bacteria without this gene. Additionally, food animals have been determined to be
the reservoir for colistin resistance microbes, from which they spread to other hosts. Due to the
adverse effects of colistin, many developed countries have prohibited its usage in animal foods, 
but developing countries are still using colistin in animal food production, thereby imposing a major risk to the public health. Therefore, there is a need for implementation of sustainable measures in livestock farms to prevent microbial infection. This review highlights the negative effects (increased resistance) of colistin consumption and emphasizes the different approaches used for detecting colistin in animal-based foods as well as the challenges associated with its detection.

Keywords: antibiotics; colistin; detection methods; food animals; multi-drug resistance

\section{Introduction}

Colistin is an antibiotic synthesized non-ribosomally by Bacillus polymyxa subspecies colistinus [1]. Colistin (polymyxin E) and polymyxin B (PMB) have high structural similarities and differ only at position six, where D-Leu is present in colistin, and D-Phe is present in PMB, as illustrated in Figure 1.

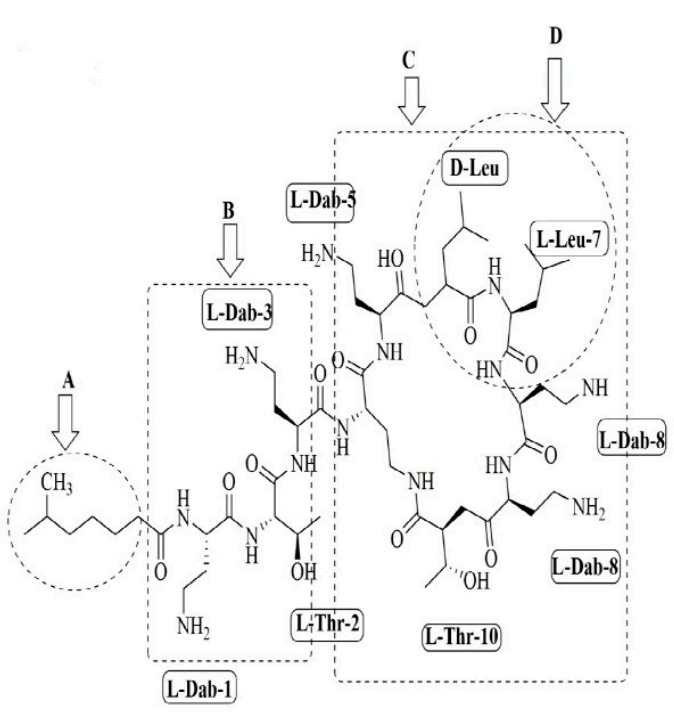

a) Polymyxin E

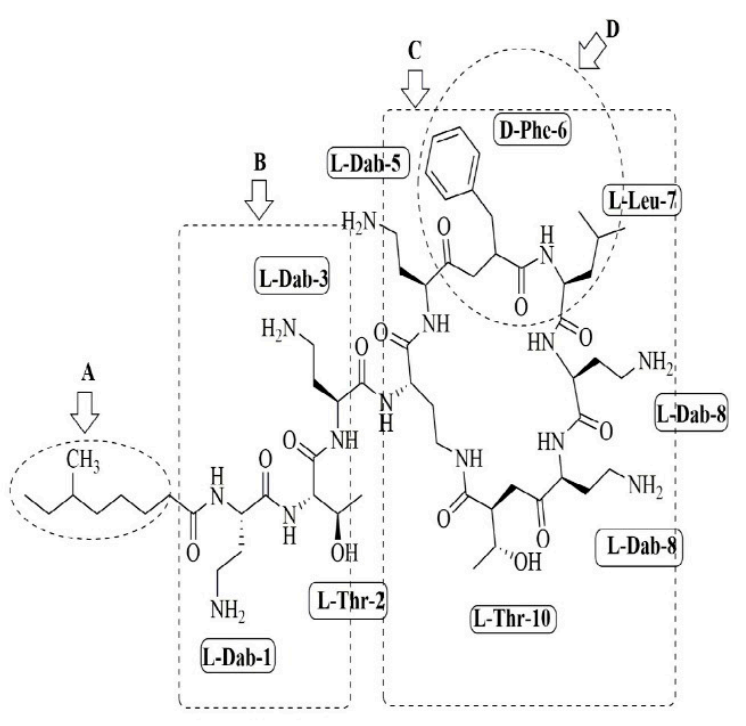

b) Polymyxin B

Figure 1. Chemical structures of polymyxin E (colistin) (a) and polymyxin B (b). The functional segments of polymyxins A: NR fatty acyl chain, B: linear tripeptide segment, C: the polar residues of the heptapeptide, D: the hydrophobic motif within the heptapeptide ring.

Colistin is effective against various Gram-negative bacteria such as Acinetobacter baumanni, Enterobactercloacae, Klebsiella pneumonia, and Pseudomonas aeruginosa [1,2]. The mechanism of action of colistin involves interactions with the outer membrane of the organism, especially lipopolysaccharide molecules, which causes displacement of calcium and magnesium ions and destabilizes the outer membrane. This destabilization of the outer membrane causes the leakage of cell content and leads to cell senescence [3,4]. During the 1970s, colistin was discontinued for clinical application as it was associated with neurotoxicity, nephrotoxicity, and other ailments [5,6]. Recently, colistin was reappraised and is being used as a last-line treatment against Gram-negative bacterial infections [6].

The extensive use of antibiotics for treating human infections caused by multidrug-resistant or highly drug-resistant Enterobacteriaceae is threatening the efficacy of colistin [7]. Furthermore, this has led to development of colistin resistance mediated by the transposable and plasmid-borne mcr genes that have been reported worldwide in Enterobacteriaceae from both humans and food-producing animals' samples. Salmonella enteric serovar infantis is one of the leading serovars among the top five Salmonella serovars involved in human infections in Europe [8]. It is most frequently detected in broilers (45.6\%) and broiler meat $(47.4 \%)$, as compared with other meats, which may be complicated by the substantial 
spread of multi-drug resistant (MDR) strains and extended spectrum beta-lactamase (ESBL)-producing S. infantis infections. According to recent reports in Switzerland and the United States, the presence of a conjugative pESI (plasmid emerging from Salmonella infantis)-like mega plasmid(harbour the $m c r-1$ gene) was found to be a significant cause of this infection, as also reported earlier in Israel and Italy in 2014 and 2015, respectively [9-12]. The mor-1 gene was found in mussels while isolating Salmonella enteric serovar Rissen ad ST-469 in northwest Spain during 2012-2016 [13]. This review highlights the uses of colistin consumption in animal-based food, its negative effects, and different approaches and advancements used for detecting colistin in animal-based food.

\section{Colistin Use in Veterinary Medicine}

For decades, colistin has been used as an additive in livestock feed for promoting growth and treating intestinal infections [14,15] as shown in Figure 2.

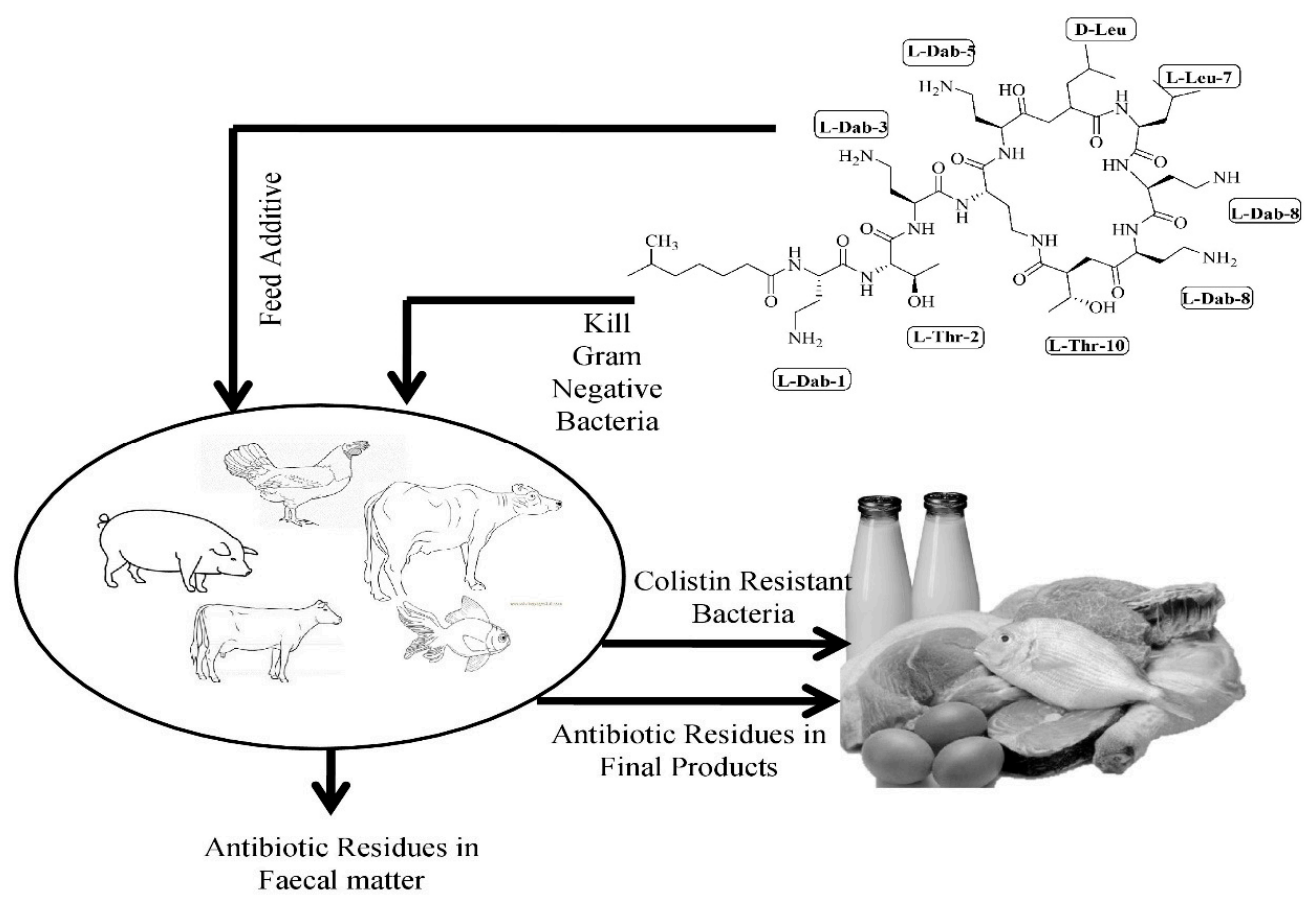

Figure 2. Graphical illustration of the spread of antibiotic-resistant bacteria as well as accumulation of colistin in animal products.

The use of colistin in animal feed and hence human consumption through the food chain has been documented in low and middle-income countries. According to a statistical analysis from 2000 to 2010, Brazil, China, India, Russia, and South Africa account for 13\% of colistin use [16]. China is a lead consumer of colistin globally, and about 2875 metric tons of colistin was consumed annually from 2011 to 2015 in this country [17]. In 2006, the European Union forbade the use of colistin in animal food to promote growth [18]. However, colistin continued to be the fifth most highly consumed drug in 2013-2015 in Europe for the treatment purpose as per the European Surveillance of Veterinary Antimicrobial Consumption Report, although no colistin drugs were marketed in Finland, Norway, or Iceland [19,20]. Additionally, some countries like USA and Canada never approved colistin usage in animal feed [19].

The recommended dosage of colistin varies according to the product and species, with 75,000 IU/kg proposed for poultry and 100,000 IU/kg for other animals like calves, rabbits and pigs; these dosages are consistent to $3.75-5 \mathrm{mg} / \mathrm{kg}$. Colistin can be administrated with complete feed, milk, water, or through injection. Approximately $0.01-0.02 \%$ of colistin is administered via milk; meanwhile, $25-50 \mathrm{mg} / \mathrm{L}$ is 
taken up by water. In feed, colistin is mixed in a range of $20-40 \mathrm{M} \mathrm{IU} / 100 \mathrm{~g}$, and $0.2 \mathrm{mg}$ of colistin is injected into 1-3-day old chicks [21].

Colistin presence in poultry occurs during the phase where mild colibacillosis is being treated, as described in earlier reports [22-24]. Its bioavailability following oral administration is very low as it does not get well absorbed from the gastrointestinal tract $[25,26]$. Common signs of colibacillosis in poultry are localized (e.g., omphalitis) or systemic (e.g., colisepticemia) that cannot be treated at the attained blood and tissue levels [27]. Drugs such as sulfonamides, tetracycline, and penicillin are more appropriate for use than administering colistin for at least seven days and at higher doses for treating mild colibacillosis [28]. Colistin is effectively used for primary diarrheal disease caused by Escherichia coli, which is rarely found in poultry but successfully used as a growth promoter [27]. Various agencies have defined the maximum residue limits (MRL) of colistin in animal-based food, as shown in Table 1.

Table 1. Maximum residue limits (MRLs) of colistin recommended in animal-based food by different regulatory agencies.

\begin{tabular}{|c|c|c|c|}
\hline Animal Species & Target Tissue & MRLs (Per Kg) & Reference \\
\hline All food producing animal spp. & Fat, muscle, liver & $150 \mu \mathrm{g}, 150 \mu \mathrm{g}, 150 \mu \mathrm{g}$ & [29] \\
\hline All food producing animal spp. & Kidney & $200 \mu \mathrm{g}$ & [29] \\
\hline All food producing animal spp. & Milk & $50 \mu \mathrm{g}$ & [29] \\
\hline All food producing animal spp. & Eggs & $300 \mu \mathrm{g}$ & [29] \\
\hline Cattle, sheep's & Fat, muscle, kidney, liver, milk & $150 \mu \mathrm{g}, 150 \mu \mathrm{g}, 200 \mu \mathrm{g}, 150 \mu \mathrm{g}, 50 \mu \mathrm{g}$ & {$[30]$} \\
\hline Pig, goat, rabbit & Fat, muscle, liver, kidney & $150 \mu \mathrm{g}, 150 \mu \mathrm{g}, 150 \mu \mathrm{g}, 200 \mu \mathrm{g}$ & [30] \\
\hline Chicken & Fat, liver, kidney, eggs & $150 \mu \mathrm{g}, 150 \mu \mathrm{g}, 200 \mu \mathrm{g}, 300 \mu \mathrm{g}$ & [30] \\
\hline Turkey & Fat, muscle, liver, kidney & $150 \mu \mathrm{g}, 150 \mu \mathrm{g}, 150 \mu \mathrm{g}, 200 \mu \mathrm{g}$ & [30] \\
\hline Cattle, lamb & Milk & $50 \mu \mathrm{g}$ & [31] \\
\hline Cattle, lamb, swine, chicken, rabbit & Fat, muscle, liver, kidney & $150 \mu \mathrm{g}, 150 \mu \mathrm{g}, 150 \mu \mathrm{g}, 200 \mu \mathrm{g}$ & [31] \\
\hline Bovine & Muscle, liver, kidney & $150 \mu \mathrm{g}, 150 \mu \mathrm{g}, 200 \mu \mathrm{g}$ & [32] \\
\hline Porcine & Liver, kidney & $150 \mu \mathrm{g}, 200 \mu \mathrm{g}$ & [32] \\
\hline Poultry & Muscle, liver, kidney & $150 \mu \mathrm{g}, 150 \mu \mathrm{g}, 200 \mu \mathrm{g}$ & [32] \\
\hline Pig, cattle, chicken & Muscle, fat, liver & $150 \mu \mathrm{g}$ & [33] \\
\hline Cattle & Milk & $50 \mu \mathrm{g}$ & [33] \\
\hline Pig, cattle, chicken & Kidney & $200 \mu \mathrm{g}$ & [33] \\
\hline
\end{tabular}

\section{Dosing Regimen of Colistin in Animals}

Colistin use varies by the type of livestock used as animal-based food. Milk-fed calves were injected with $5 \mathrm{mg} / \mathrm{kg}$ of colistin sulfate (CS) (commercially available form), and $16 \mu \mathrm{g} / \mathrm{mL}$ was recorded in peak serum concentration analysis. On the other hand, $1.3 \mathrm{~L} / \mathrm{kg}$ of colistin was used for volume distribution and $3.4 \mathrm{~mL} / \mathrm{min} / \mathrm{kg}$ for renal clearance with an excretion half-life of 5-6 $\mathrm{h}[34,35]$. The serum of dairy cows and calves showed the persistence of colistimethate sodium when it was injected intramuscularly. The highest value $(60 \mathrm{IU} / \mathrm{mL})$ of colistin concentration in the serum of cows was recorded within $3 \mathrm{~h}$ after the administration of colistin. The highest peak of serum concentration in calves was recorded $1-2 \mathrm{~h}$ after colistin administration, and the calculated half-life was found to be two-fold more in cows as compared to calves. The concentration of colistin is very low in milk and sometimes is found to be in the detectable range after a second milking, whereas the well-diffused microbiological method shows no residue in calves [36]. Another study revealed that peak colistin concentration can be measured within $2 \mathrm{~h}$ of dosing in serum and traces of it can be measured up to $6 \mathrm{~h}$ after intravenous administration, as the detectable range was found to be $0.1-1 \mu \mathrm{g} / \mathrm{mL}$ in serum. There is no detectable limit for oral administration of colistin [37]. The administration of colistimethate sodium intramuscularly increases serum concentration as compared to colistin sulfate for dosages of 3.5 and $7.5 \mathrm{mg} / \mathrm{kg}$, respectively. This further reveals that serum protein has a binding affinity towards colistin sulfate in comparison to colistimethate in ewes [38]. Similar results have also been recorded for dogs [39]. The colistin binding with plasma protein was found to be $40 \%$ for cattle. In chickens, after oral injection of $50 \mathrm{mg} / \mathrm{kg}$ of colistin, the maximum concentrations of 5.7 and $10.2 \mu \mathrm{g} / \mathrm{mL}$, respectively, were detected in bile and serum after $2 \mathrm{~h}$.

Sato et al. [40] also conducted experiments on pigs using two different doses of colistin, i.e., 25 and $50 \mathrm{mg} / \mathrm{kg}$. They reported peak serum concentrations of $1.0 \mathrm{and} 8.3 \mu \mathrm{g} / \mathrm{mL}$ after $1 \mathrm{~h}$ of administration of 
colistin in two different doses, as the sample is untraceable in later stages. The maximum concentrations of 4.0 and $1.0 \mu \mathrm{g} / \mathrm{mL}$ in bile and serum of pigs were detected respectively after oral administration of $25 \mathrm{mg} / \mathrm{kg}$ of colistin [40]. Another study reported the intractability of colistin in the serum of gnotobiotic piglets which were fed $40 \mathrm{mg} / \mathrm{kg}$ of colistin in sterilized milk [41]. The research was conducted on pigs to assess the effect of oral dosages of 2.5 and $5.0 \mathrm{mg} / \mathrm{kg}$ along with a $2.5 \mathrm{mg} / \mathrm{kg}$ intravenous dose, and it was observed that the peak concentration of plasma was attained after $30 \mathrm{~min}$ of administration and the half-life for both the doses was found to be $4.5 \mathrm{~h}$ with a clearance rate of about $3 \mathrm{~mL} / \mathrm{kg} / \mathrm{min}$ [42]. CS concentrations were very difficult to calculate in the plasma of the healthy pigs after oral administration, despite the use of exact and accurate analytical methods $[43,44]$. A concurrent oral challenge of pigs with an Enterotoxigenic Escherichia coli (ETEC) was done. The F4 strain did not increase CS intestinal absorption in a subclinical induction model of post-weaning diarrhoea (PWD) [44]. However, CS concentrations in plasma were higher in pigs with clinical post-weaning diarrhoea following an experimental oral challenge as compared to the unchallenged pigs [45]. These studies revealed the low absorption of CS through the gastro-intestinal tract of pigs even in infected animals and corroborate the involvement of oral CS administration in increasing colistin resistance by exerting selection pressure (due to antibiotic) on the intestinal flora of pigs [46].

\section{Negative Consequences of Colistin Consumption}

Until 2015, colistin resistance in the Enterobacteriaceae family was believed to be generated via chromosomal mechanisms which modified the lipopolysaccharide (LPS) layer by adding 2-aminoethanol, phosphoethanolamine (PetN) (a derivative of 2-aminoethanol), or other efflux pumps or by forming capsules in these microorganisms [47]. Lipid A modification of LPS can be associated with mutations triggering the activation of two-component systems including PmrA/PmrB and PhoP/PhoQ or inactivating the $m g r B$ gene, which induces negative feedback of the PhoP/PhoQ system in Gram-negative species. In Escherichia coli, the etk and $m g r R$ genes have been found to confer resistance against colistin [48]. In 2015, Chinese researchers studied the colistin-resistant strains of bacteria and reported the presence of the $\mathrm{mcr}-1$ gene, which can transfer itself from one bacterial strain to another [49]. The mcr-1 gene encodes a phosphoethanolamine transferase, which catalyzes the addition of phosphoethanolamine (a cationic molecule) to lipid A of LPS, which changes the charge of the cell membrane, and as result colistin (cationic) is unable to bind and triggers the lysis of the cell membrane [50]. The PCR-based screening has enabled researchers to find $m c r-2$ to $m c r-8$ genes (plasmid-mediated colistin-resistant genes) and revealed theprevalence of the $\mathrm{mcr}$-2 gene in Escherichia coli strains isolated from bovine and porcine [51-57]. Moreover, $m c r-2$ to $m c r-8$ genes share $44-77 \%$ similarity with $m c r-1$, and the gene products synthesized by them have $32-83 \%$ similarity to the amino acid sequence of $m c r-1$. A list of plasmid-borne $m c r-1$ in bacteria isolated from animal-based food is shown in Table 2. 
Table 2. Isolation of colistin-resistant microbes from animal-based food in different countries.

\begin{tabular}{|c|c|c|c|c|c|c|}
\hline Country & Type of Animals & Type of Samples & Sample Size & Type of Microbes & Detection Basis & Reference \\
\hline Nepal & Healthy chickens & Cloacal swabs & 324 & Escherichia coli & $m c r-1$ & [58] \\
\hline \multirow{4}{*}{ China } & Healthy chickens & Lung, spleen & 644 & \multirow{4}{*}{ Escherichia coli } & \multirow{4}{*}{$m c r-1$} & \multirow{4}{*}{ [59] } \\
\hline & Pigs & Liver & 113 & & & \\
\hline & Cows & Milk & 61 & & & \\
\hline & Ducks & Liver & 44 & & & \\
\hline \multirow{4}{*}{ Iran } & Healthy broilers & \multirow{4}{*}{ Cloacal swabs } & 503 & \multirow{4}{*}{ Klebsiella pneumoniae } & \multirow{4}{*}{$m c r-1,2,3,4$} & \multirow{4}{*}{ [60] } \\
\hline & Dead broilers & & 388 & & & \\
\hline & Dead lying hens & & 30 & & & \\
\hline & Dead turkeys & & 23 & & & \\
\hline \multirow{2}{*}{ Vietnam } & Healthy chickens & \multirow{2}{*}{ Cloacal swabs } & \multirow{2}{*}{ NS } & \multirow{2}{*}{ Escherichia coli } & \multirow{2}{*}{$m c r-1$} & \multirow{2}{*}[61]{} \\
\hline & Pigs & & & & & \\
\hline \multirow{3}{*}{ Brazil } & \multirow{3}{*}{ Healthy chickens } & Breast & 20 & \multirow{3}{*}{ Escherichia coli } & \multirow{3}{*}{$m c r-1$} & \multirow{3}{*}[62]{} \\
\hline & & Thigh & 20 & & & \\
\hline & & Liver & 1 & & & \\
\hline Denmark & Chicken meat & ND & NS & Escherichia coli & $m c r-1$ & [63] \\
\hline \multirow{3}{*}{ Spain } & \multirow{2}{*}{ Swine } & Lymph node & \multirow{2}{*}{ NS } & \multirow{2}{*}{ Salmonella enterica, Escherichia coli } & & \multirow{3}{*}[64]{} \\
\hline & & Faeces & & & $m c r-1$ & \\
\hline & Turkey & Faeces & NS & Escherichia coli & & \\
\hline Switzerland & Chicken meat & $\mathrm{ND}$ & 6 & Escherichia coli & $m c r-1$ & [65] \\
\hline \multirow[b]{2}{*}{ Germany } & Healthy chicken & Drumsticks & 500 & Cirobacter freundii, Klebsiella oxytoca, Pantoea agglomerans & \multirow{2}{*}{ Disc diffusion } & \multirow{2}{*}{ [66] } \\
\hline & Pork & Belly & 500 & Escherichia coli, Klebsiella oxytoca & & \\
\hline Japan & Diseased swine & ND & NS & Escherichia coli & $m c r-1$ & [67] \\
\hline Algeria & Healthy chickens & ND & NS & Escherichia coli & Disc diffusion & [68] \\
\hline \multirow{5}{*}{ Taiwan } & Diseased Chickens & \multirow{5}{*}{ ND } & 450 & \multirow{5}{*}{ Salmonella spp. } & & \\
\hline & Pigs & & 279 & & & \\
\hline & Ducks & & 206 & & $m c r-1$ & [69] \\
\hline & Turkeys & & 170 & & & \\
\hline & Geese & & 88 & & & \\
\hline Great Britain & Diseased pigs & Small intestine & 3 & Escherichia coli, Salmonella typhimurium & $m c r-1$ & [70] \\
\hline
\end{tabular}


Table 2. Cont.

\begin{tabular}{|c|c|c|c|c|c|c|}
\hline Country & Type of Animals & Type of Samples & Sample Size & Type of Microbes & Detection Basis & Reference \\
\hline \multirow{3}{*}{ Great Britain } & Healthy Pigs & Cecums & 2509 & \multirow{3}{*}{ Escherichia coli } & \multirow{3}{*}{ Disc diffusion } & \multirow{3}{*}{ [71] } \\
\hline & Cattle & Distal rectums & 891 & & & \\
\hline & Sheep & Distal rectums & 973 & & & \\
\hline Italy & Diseased pigs & Rectal swabs, faeces, intestines & NS & Escherichia coli & Disc diffusion, $m c r-1$ & [72] \\
\hline Great Britain & Healthy pigs & Cecal contents & NS & Moraxella spp. & MIC and $m c r-1,2$ & [52] \\
\hline \multirow{5}{*}{ France } & \multirow{5}{*}{ Diseased pigs } & Intestinal & 63 & \multirow{5}{*}{ Escherichia coli } & \multirow{5}{*}{ Disc diffusion } & \multirow{5}{*}{ [48] } \\
\hline & & Septicemia & 2 & & & \\
\hline & & Nervous system & 1 & & & \\
\hline & & Lymph node & 1 & & & \\
\hline & & Urine & 1 & & & \\
\hline \multirow{3}{*}{ Botswana } & \multirow{3}{*}{ Beef } & Meat cubes & 134 & \multirow{3}{*}{ Escherichia coli O157: H7 } & \multirow{3}{*}{ Disc diffusion } & \multirow{3}{*}[73]{} \\
\hline & & Minced meat & 133 & & & \\
\hline & & Fresh sausages & 133 & & & \\
\hline India & Poultry & ND & NS & Salmonella spp. & Disc diffusion & [74] \\
\hline \multirow{3}{*}{ India } & Chickens & Faecal, cecal & 434 & \multirow{3}{*}{ Salmonella enterica } & \multirow{3}{*}{ Disc diffusion } & \multirow{3}{*}{ [75] } \\
\hline & Ducks & Faecal & 38 & & & \\
\hline & Emus & Faecal & 35 & & & \\
\hline
\end{tabular}

ND—not defined; NS—not specified. 
Colistin is an ancient drug that was banned because of its nephrotoxicity and neurotoxicity activity in humans; however, it was reintroduced to treat carbapenem resistance in Gram-negative bacteria (Supplementary Table S1). Unfortunately, colistin resistance mechanisms have now been documented in Enterobacteriaceae strains capable of producing carbapenemase, making them resistant to both classes of drugs and a global health concern [76-79].

In 2016, the government of China banned the use of colistin as a food additive for livestock. China alone was using 8000 tons of colistin per annum, whereas global production was 12,000 tons per annum. Despite this ban, agrichemical companies in China were the leading colistin producer and tons of colistin were exported to countries like India, South Korea, and Vietnam [80]. In India, five animal pharmaceutical companies advertise products containing colistin for promoting growth or use for metaphylactic purposes. As per the investigation carried out by the Bureau of Investigative Journalism of London, chickens raised in India are heavily dosed with strong antibiotics. Venky's, the chief supplier of chicken products in India, has been reported to use the antibiotic colistin for therapeutic purposes [81]. These practices are highly unsafe as drug-resistance is very common, and about $57 \%$ of Gram-negative bacteria in India are carbapenem-resistant. Therefore, India depends on colistin for treating acute infections in humans (in contrast, resistance to carbapenem in Klebsiella pneumoniae is less than $1 \%$ in the United Kingdom) [80].

The Government of India did take the initiative to ban the usage of colistin antibiotics as a growth supplement, but this initiative has not yet been associated with any regulatory body. Now the Food Safety and Standards Authority of India (FSSAI) claims to have fixed the tolerance level of antibiotics in food-based items, and they have also revised current standards governing toxics, residues, and contaminants under the 2011 regulations [82]. Finally, in 2019, the FSSAI implemented a complete ban on colistin use in India [83].

\section{Routine Methods for Colistin Detection in Animals and Its Associated Challenges}

Colistin, being a polar drug, forms a strong bond with phospholipids or proteins, which makes drug extraction a more complicated process in tissues [84]. Hence, limited systems have been created and are available to find colistin antibiotics in food, as shown in Table 3. 
Table 3. Different conventional methods used for the detection of colistin in animal-based food.

\begin{tabular}{|c|c|c|c|c|c|c|c|c|}
\hline \multirow{2}{*}{ Country } & \multirow{2}{*}{ Sample } & \multirow{2}{*}{ Method Used } & \multicolumn{4}{|c|}{ Chromatography Conditions Used } & \multirow{2}{*}{ Detection Limit } & \multirow{2}{*}{ Reference } \\
\hline & & & Model & Column & Solvent & Flow Rate & & \\
\hline China & Spiked bovine milk & HPLC-MS/MS & $\begin{array}{l}\text { An HPLC (Hewlett-Packard HP } \\
1100 \text { series, Rockville, MD, USA) } \\
\text { integrated system consisting of a } \\
100 \text {-well auto-sampler, a } 100 \mu \mathrm{L} \\
\text { sample loop, a degasser, } \\
\text { a quaternary pump and a } \\
\text { thermostated column oven set at } \\
25^{\circ} \mathrm{C} \text { was used }\end{array}$ & $\begin{array}{l}\text { Chromatographic separation was } \\
\text { performed in a } 250 \mathrm{~mm} \times 2.1 \mathrm{~mm} \text {, } \\
5 \mu \mathrm{m} \text { Alltima } \mathrm{C}_{18} \text { separation } \\
\text { column (Alltech, Deerfield, MA, } \\
\text { USA) and a corresponding } \mathrm{C}_{18} \\
\text { guard column }(7.5 \mathrm{~mm} \times 4.6 \mathrm{~mm})\end{array}$ & $\begin{array}{c}\text { Mobile phase A: } 0.1 \% \text { formic acid in } \\
\text { acetonitrile and mobile phase B: saturated } \\
\text { ammonium formate:formic } \\
\text { acid:acetonitrile:water }(1: 5: 50: 950, v / v / v / v)\end{array}$ & $\begin{array}{l}\text { Flow rate of } 0.2 \mathrm{~mL} \mathrm{~min}^{-1} \text { under a } \\
\text { gradient elution program } \\
\text { comprised of two mobile phases }\end{array}$ & $50 \mu g / \mathrm{Kg}$ & [85] \\
\hline China & Spiked fishery products & UPLC-MS/MS & $\begin{array}{c}\text { A UPLC-MS/MS system } \\
\text { comprised an Acquity ULLC } \\
\text { system connected online with a } \\
\text { Quattro Premier tandem mass } \\
\text { spectrometer (Waters, Milford, } \\
\text { MA, USA) } \\
\end{array}$ & $\begin{array}{l}\text { The column used was an } \\
\text { ACQUITYTM BEH } C_{18} \text { reversed } \\
\text { phase column }(2.1 \mathrm{~mm} \times 100 \mathrm{~mm}, \\
1.7 \mu \mathrm{m} \text { particle size) maintained at } \\
40^{\circ} \mathrm{C}\end{array}$ & $\begin{array}{l}\text { Mobile phase was } 0.2 \% \text { formic acid in } \\
\text { acetonitrile and } 0.2 \% \text { formic acid in water }\end{array}$ & $\begin{array}{l}\text { Flow rate and temperature of the } \\
\text { drying gas }\left(\mathrm{N}_{2}\right) \text { were } 750 \mathrm{~L} \mathrm{~h}^{-1} \\
\text { and } 350^{\circ} \mathrm{C} \text {, respectively. The cone } \\
\text { gas flow }\left(\mathrm{N}_{2}\right) \text { was } 50 \mathrm{~L} \mathrm{~h}^{-1}\end{array}$ & $\begin{array}{l}10 \mu \mathrm{gg} / \mathrm{Kg} \text { (colistin A), } \\
40 \mu \mathrm{g} / \mathrm{Kg} \text { (colistin B) }\end{array}$ & [86] \\
\hline Hungary & Spiked pig feeds & $\begin{array}{l}\text { HPLC-fluorescence } \\
\text { detector }\end{array}$ & $\begin{array}{l}\text { JASCO PU-980 high pressure } \\
\text { pump (JASCO, Kyoto, Japan) }\end{array}$ & $\begin{array}{l}\text { A TSK ODS 120T column }(150 \times \\
4.6 \mathrm{mmID}, 5 \mu \mathrm{m}) \text { was used with an } \\
\text { injection volume of } 25 \mu \mathrm{L}\end{array}$ & $\begin{array}{c}\text { Mobile phase was } 22: 78 \mathrm{v} / \mathrm{w} \text { acetonitrile- } 50 \\
\mathrm{mM} \text { sodium sulfate, } 20 \mathrm{mM} \\
\text { orthophosphoric acid, } 25 \mathrm{mM} \text { triethylamine }\end{array}$ & $\begin{array}{l}\text { Flow rate of the mobile phase and } \\
\text { post-column reagent were } 1.5 \text { and } \\
1.0 \mathrm{~mL} \mathrm{~min}^{-1} \text {, respectively }\end{array}$ & $20 \mathrm{mg} / \mathrm{Kg}$ & [87] \\
\hline Spain & Spiked animal feeds & $\begin{array}{l}\text { HPLC-fluorescence } \\
\text { detector }\end{array}$ & $\begin{array}{l}\text { ee Thermo HPLC system equipped } \\
\text { with a P200 gradient pump }\end{array}$ & $\begin{array}{c}\text { Analytical column }(150 \times 4.6 \mathrm{~mm} \\
\text { i.d.) used was packed with } \\
\text { Ultracarb } 5 \mu \mathrm{m} \text { ODS } 30 \% \mathrm{C} \text {. Guard } \\
\text { columns }(50 \times 4.6 \mathrm{~mm} \text { i.d.) were } \\
\text { packed with dry } 40 \mu \mathrm{m} \text { Pelliguard } \\
\text { LC- } 18\end{array}$ & $\begin{array}{l}\text { Mobile phases with methanol and } \\
\text { acetonitile }\end{array}$ & Flow of $1.5 \mathrm{~mL}$ & $5 \mathrm{mg} / \mathrm{Kg}$ & [88] \\
\hline France & $\begin{array}{l}\text { Spiked bovine milk and } \\
\text { tissues (muscle, liver, } \\
\text { kidney, fat) }\end{array}$ & HPLC-MS & $\begin{array}{l}\text { The HPLC system consisted of a } \\
\text { solvent delivery pump (model } \\
\text { P2000, Thermo Separation } \\
\text { Products, Les Ulis, France), } \\
\text { an injection valve (model } 7725 \mathrm{i} \text {, } \\
\text { Rheodyne, Cotati, CA, USA) }\end{array}$ & $\begin{array}{c}\text { An analytical column }(125 \times 4 \mathrm{~mm} \\
\text { i.d.) pre-packed with } 5 \\
\mu \text { Nucleosil } \mathrm{C}_{18} \text { (Macherey-Nagel, } \\
\text { Düren, Germany) }\end{array}$ & $\begin{array}{l}\text { Mobile phase was acetonitrile and a } 0.035 \mathrm{M} \\
\text { triethylamine solution adjusted to pH } 2.5 \\
\text { with phosphoric acid and mixed in 17:83 } \\
(v / v) \text { proportions }\end{array}$ & The flow rate was $1.5 \mathrm{~mL} / \mathrm{min}$ & $\begin{array}{l}25 \mu \mathrm{\mu g} / \mathrm{L} \text { (milk), } 100 \mu \mathrm{\mu g} / \mathrm{Kg} \\
\text { (tissues) }\end{array}$ & [89] \\
\hline China & $\begin{array}{l}\text { Swine liver, chicken eggs, } \\
\text { feed, swine muscles, } \\
\text { chicken muscles, bovine } \\
\text { muscles, sheep muscles, } \\
\text { bovine raw milk }\end{array}$ & UHPLC-MS/MS & $\begin{array}{l}\text { An Acquity ultra-performance } \\
\text { liquid chromatography system } \\
\text { (Waters, Milford, MA, USA) }\end{array}$ & $\begin{array}{c}\text { An Acquity BEH C } 18 \text { column } \\
(50 \mathrm{~mm} \times 2.1 \mathrm{~mm} \text { i.d., } 1.7 \mu \mathrm{mm} \\
\text { particle size) (Waters, Milford, MA, } \\
\text { USA) }\end{array}$ & $\begin{array}{l}\text { Mobile phases comprised of } 0.5 \% \text { formic } \\
\text { acid in water (solvent A) and } 0.5 \% \text { formic } \\
\text { acid in acetonitrile (solvent B) }\end{array}$ & $\begin{array}{c}\text { Flow rate was } 0.4 \mathrm{~mL} / \mathrm{min} \text { with the } \\
\text { following gradient program: } \\
0-0.5 \mathrm{~min}, 95 \% \mathrm{~A} ; 0.5-3.0 \mathrm{~min} \\
95-50 \% \mathrm{~A} ; 3.0-4.0 \mathrm{~min}, 50-5 \% \mathrm{~A} ; \\
4.0-4.1 \mathrm{~min}, 5-95 \% \mathrm{~A} ; 4.1-5.5 \mathrm{~min} \\
95 \% \mathrm{~A}\end{array}$ & $5-30 \mu g / \mathrm{Kg}$ & [90] \\
\hline Belgium & Spiked swine manure & UHPLC-MS/MS & $\begin{array}{l}\text { An Acquity UPLC H-class system } \\
\text { (Waters, Milford, MA, USA) }\end{array}$ & $\begin{array}{l}\text { Reversed-phase Kinetex } \mathrm{C}_{18} \\
\text { column (100 mm } \times 2.1 \mathrm{~mm} \text { i.d., } \\
1.7 \mu \mathrm{m} \text { ) with a SecurityGuard Ultra } \\
\text { guard cartridge system } \\
\text { (Phenomenex, Utrecht, } \\
\text { The Netherlands) }\end{array}$ & 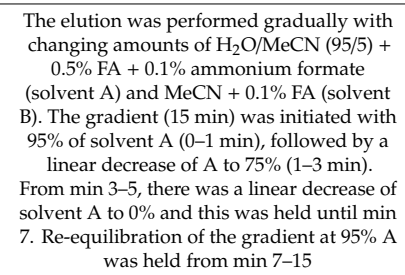 & Flow at $400 \mu \mathrm{L} / \mathrm{min}$ & $\begin{array}{c}20.2 \mu \mathrm{\mu g} / \mathrm{Kg} \text { (colistin A), } \\
15 \mu \mathrm{\mu g} / \mathrm{Kg} \text { (colistin B) }\end{array}$ & [91] \\
\hline
\end{tabular}


Table 3. Cont

\begin{tabular}{|c|c|c|c|c|c|c|c|c|}
\hline \multirow{2}{*}{ Country } & \multirow{2}{*}{ Sample } & \multirow{2}{*}{ Method Used } & \multicolumn{4}{|c|}{ Chromatography Conditions Used } & \multirow{2}{*}{ Detection Limit } & \multirow{2}{*}{ Reference } \\
\hline & & & Model & Column & $\begin{array}{rr}\text { Solvent } \\
\end{array}$ & Flow Rate & & \\
\hline China & $\begin{array}{l}\text { Spiked swine and poultry } \\
\text { feeds }\end{array}$ & UHPLC-MS/MS & $\begin{array}{l}\text { LC-MS/MS system (Thermo } \\
\text { Electron Corp., Wyman, Waltham, } \\
\text { MA, USA) consisting of a Finnigan } \\
\text { Surveyor Plus system with an } \\
\text { online degasser, a Surveyor } \\
\text { autosampler and a TSQ Quantum } \\
\text { triple quadrupole mass } \\
\text { spectrometer equipped with an } \\
\text { electrospray interface operating in } \\
\text { the positive mode (ESI+) }\end{array}$ & $\begin{array}{c}\text { Separation was performed on } \\
150 \mathrm{~mm} \times 2.1 \mathrm{~mm}, 5 \mu \mathrm{m} \text { Hypersil } \\
\text { Gold } \mathrm{C}_{18} \text { analytical columns } \\
\text { (Thermo Electron Corporation, } \\
\text { Waltham, MA, USA) }\end{array}$ & $\begin{array}{l}\text { Mobile phase A consist formic acid in water } \\
\text { and mobile phase B formic acid in ACN }\end{array}$ & Flow-rate of $0.2 \mathrm{~mL} \mathrm{~min}^{-1}$ & $\begin{array}{l}27.5 \mu \mathrm{\mu g} / \mathrm{Kg} \text { (colistin A), } \\
25.7 \mu \mathrm{\mu g} / \mathrm{Kg} \text { (colistin B) }\end{array}$ & [92] \\
\hline China & $\begin{array}{l}\text { Spiked piglet premix, } \\
\text { pig feed additive, poultry } \\
\text { complete feed, } \\
\text { pig complete feed and } \\
\text { fattening pig premix }\end{array}$ & UHPLC-MS/MS & $\begin{array}{l}\text { Shimadzu liquid chromatography } \\
\text { system (Shimadzu, Kyoto, Japan) }\end{array}$ & $\begin{array}{l}\text { Separations were carried out on a } \\
\text { Phenomenex Kinetex Biphenyl } \\
\text { column }(50 \mathrm{~mm} \times 2.1 \mathrm{~mm} \text { i.d., } \\
2.6 \mu \mathrm{m} \text { particle size, Phenomenex, } \\
\text { Torrance, CA, USA) }\end{array}$ & $\begin{array}{c}\text { Mobile phase consisted of } 0.1 \% \mathrm{FA} \text { in } \mathrm{ACN} \\
\text { solution (A) and } 0.1 \% \mathrm{FA} \text { in water solution } \\
\text { (B) with the following gradient elution } \\
\text { program: } 0 \mathrm{~min}, 6 \% \mathrm{~A} ; 2 \mathrm{~m} \text { min, } 6 \% \mathrm{~A} ; 5 \mathrm{~min} \text {, } \\
40 \% \mathrm{~A} ; 14 \mathrm{~min}, 70 \% \mathrm{~A} ; 14.1 \mathrm{~min}, 6 \% \mathrm{~A} ; \\
18 \mathrm{~min}, 6 \% \mathrm{~A}\end{array}$ & Flow rate of $0.2 \mathrm{~mL} / \mathrm{min}$. & $\begin{array}{l}5-20 \mu g / K g \text { (colistin A), } \\
\text { and (colistin B) }\end{array}$ & [93] \\
\hline Canada & Spiked chicken muscle & UPLC-MS/MS & $\begin{array}{l}\text { Waters Acquity UPLC interfaced } \\
\text { to a Waters Micromass triple } \\
\text { quadrupole Premier mass } \\
\text { spectrometer equipped with an ESI } \\
\text { source and controlled by } \\
\text { MassLynx 4.1 software(Waters, } \\
\text { Milford, MA, USA) }\end{array}$ & $\begin{array}{l}\text { Poroshell } 120,100 \times 2.1 \mathrm{~mm} \text { id, } \\
2.7 \text {, }(\text { Agilent Technologies, } \\
\text { Mississauga, ON Canada) }\end{array}$ & $\begin{array}{l}\text { Mobile phase A ( } 0.1 \% \text { formic acid in } \\
\text { water)and mobile phase B (methanol) }\end{array}$ & Flow rate of $0.40 \mathrm{~mL} / \mathrm{min}$ & $\begin{array}{l}39 \mu \mathrm{gg} / \mathrm{Kg} \text { (colistin A), } \\
50 \mu \mathrm{g} / \mathrm{Kg} \text { (colistin B) }\end{array}$ & [94] \\
\hline Italy & Spiked bovine milk, meat & HPLC-MS & $\begin{array}{l}\text { Thermo Ultimate } 3000 \text { High } \\
\text { Performance Liquid } \\
\text { Chromatography system (Thermo } \\
\text { Scientific, San Jose, CA, USA) }\end{array}$ & $\begin{array}{l}\text { InfinityLab Poroshell } 120 \text { HILIC } \\
\text { column }(100 \times 2.1 \mathrm{~mm} ; 2.7 \mu \mathrm{m}, \\
\text { Agilent Technologies, Santa Clara, } \\
\text { CA, USA) connected with the } \\
\text { InfinityLab Poroshell } 120 \mathrm{HILICC} \\
\text { guard column }(5 \times 2.1 \mathrm{~mm}, 2.7 \mu \mathrm{m})\end{array}$ & 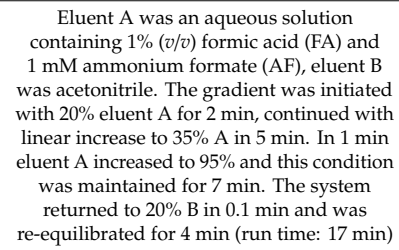 & Flow rate was $0.25 \mathrm{~mL} \mathrm{~min}^{-1}$ & $33 \mu \mathrm{g} / \mathrm{Kg}$ & [95] \\
\hline Hong Kong & $\begin{array}{l}\text { Spiked bovine milk and } \\
\text { tissues }\end{array}$ & HPLC-MS/MS & $\begin{array}{c}\text { An integrated HPLC system } \\
\text { (Hewlett-Packard HP 1100series, } \\
\text { Rockville, MD, USA) consisting of } \\
\text { a 100-well autosampler, a degasser, } \\
\text { two-channel binary pump, and } \\
\text { atemperature control oven (set at } \\
25^{\circ} \mathrm{C} \text { ), and interfaced with a TSQ } \\
\text { Quantum Discovery mass } \\
\text { spectrometer (Thermo-Finnigan, } \\
\text { San Jose, CA, USA) }\end{array}$ & $\begin{array}{c}150 \mathrm{~mm} \times 2.1 \mathrm{~mm}, 5 \mu \mathrm{m} \\
\text { Phenomenex Luna } \mathrm{C}_{18} \text { analytical } \\
\text { column (Torrance, CA, USA) } \\
\text { connected to a } 7.5 \mathrm{~mm} \times 4.6 \mathrm{~mm} \\
\text { Alltech Alltima } \mathrm{C}_{18} \text { guard column } \\
\text { (Deerfield, IL, USA) }\end{array}$ & $\begin{array}{l}\text { Mobile phases, which were comprised of a } \\
\text { mixture of (A) } 0.1 \% \text { formic acid in water and } \\
\text { (B) } 0.1 \% \text { formic acid in acetonitrile, } \\
\text { were delivered under a gradient elution } \\
\text { program (0-4 min: } 95 \% \mathrm{~A}, 5 \% \mathrm{~B} ; 4-8 \mathrm{~min}: \\
30 \% \mathrm{~A}, 70 \% \mathrm{~B} \text { and held for } 4 \text { min; } 12 \mathrm{~min}: \\
95 \% \mathrm{~A}, 5 \% \mathrm{~B} \text { and held for } 3 \text { min to restore } \\
\text { initial conditions before the next injection }\end{array}$ & Flow-rate of $0.25 \mathrm{~mL} \mathrm{~min}{ }^{-1}$ & $\begin{array}{l}1-16 \mu g / K g \text { (colistin A), } \\
6-14 \mu g / \mathrm{Kg} \text { (colistin B) }\end{array}$ & [96] \\
\hline
\end{tabular}


Table 3. Cont

\begin{tabular}{|c|c|c|c|c|c|c|c|c|}
\hline \multirow{2}{*}{ Country } & \multirow{2}{*}{ Sample } & \multirow[b]{2}{*}{ Method Used } & \multicolumn{4}{|c|}{ Chromatography Conditions Used } & \multirow[b]{2}{*}{ Detection Limit } & \multirow[b]{2}{*}{ Referenc } \\
\hline & & & Model & Column & Solvent & Flow Rate & & \\
\hline Switzerland & $\begin{array}{l}\text { Spiked bovine liver, kidney, } \\
\text { muscle, egg, milk }\end{array}$ & UHPLC-MS/MS & $\begin{array}{l}\text { Acquity system (sample and } \\
\text { solvent manager) from Waters } \\
\text { (Millford, MA, USA) }\end{array}$ & $\begin{array}{l}\text { Kinetex } \mathrm{C}_{18}, 2.1 \times 150 \mathrm{~mm} \times \\
\text { 2.6 um column with an installed } \\
\text { pre-filter (Krud-katcher), both from } \\
\text { Phenomenex (Torrance CA, USA) }\end{array}$ & $\begin{array}{l}\text { Mobile phase A: } 50 \mathrm{~mL} \text { acetonitrile, } 3 \mathrm{~mL} \text { of } \\
\text { formic acid and } 0.1 \mathrm{~mL} \text { of trifluoroacetic } \\
\text { acid were transferred into a } 1000 \mathrm{~mL} \\
\text { volumetricflask and diluteded to volume with } \\
\text { purified water; Mobile phase B: } 50 \mathrm{~mL} \text { of } \\
\text { purified water, } 3 \mathrm{~mL} \text { of formic acidand } 0.1 \\
\mathrm{~mL} \text { of trifluoroacetic acid were transferred } \\
\text { into a 1000-mLvolumetric flask and diluted } \\
\text { to volume with ACN }\end{array}$ & 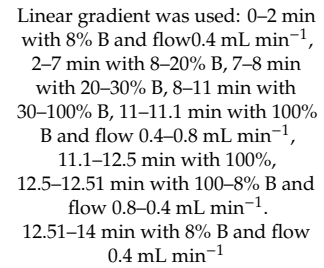 & 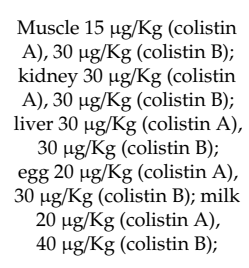 & [97] \\
\hline France & Spiked milk & $\begin{array}{l}\text { Disc diffusion } \\
\text { method (STAR } \\
\text { protocol) }\end{array}$ & NA & NA & NA & NA & $1 \mathrm{mg} / \mathrm{L}$ & [98] \\
\hline
\end{tabular}

NA—not applicable. 
Earlier, this drug was identified by adding chromophore/fluorophore groups which allow its detection with conventional LC detectors. Sin et al. [85] first published a paper for bacitracin and colistin detection using LC-MS/MS in the kidney, liver, and milk. Deproteinized milk samples were extracted with a mixture of trichloroacetic/formic acid and the presence of bacitracin and colistin in extracts was determined using a reversed-phase Alltima BDS $\mathrm{C}_{18}$ column using a gradient elution of ammonium formate buffer and $0.1 \%$ formic acid in acetonitrile at $0.2 \mathrm{~mL} \mathrm{~min}{ }^{-1}$. For identification and quantification of major components of these two polypeptides, electrospray LC-MS/MS with time scheduled multiple reaction monitoring (MRM) based upon the intensities of mass fragments from the bacitracin A at $712 \rightarrow 199$ amu and $712 \rightarrow 227$ amu and colistin A at 586 $\rightarrow 101 \mathrm{amu}, 586 \rightarrow 202$ amu and $586 \rightarrow 241$ amu were used.

An upgraded procedure for the detection of colistin B in the liver, muscle, and milk was also developed [96]. This method proved to be a fast screening and quantitative protocol for monitoring the concerned polypeptides present in food as a part of a surveillance program. $\mathrm{Xu}$ et al. [86] developed an analytical procedure for colistin A and B in fish products. In this study, the extraction of samples was done with $1.0 \mathrm{~mol} / \mathrm{L}$ of hydrochloric acid $(\mathrm{HCl})$ in methanol-water, and the sample was further purified on PLS solid-phase extraction columns. Multiple reaction monitoring was performed afterward using precursor-product ion combinations and resulted in mean recovery between $72.9 \%$ and $82.9 \%$. Kaufmann and Widmer [97] also reported a multi-residue method capable of detecting five polymyxins with selective and acceptable recoveries for all compounds. In this study, using a modern core-shell column with an eluent with trifluoroacetic acid, formic acid and acetonitrile resulted in chromatographically well-resolved analyte peaks. Boison et al. [94] further improved this technique and were able to detect seven polymyxins in chicken muscle. This process does not use ion-pairing reagents during the mobile phase, which permits the use of the same instrument again to perform different analyses, whereas the use of ion-pairing reagents requires effective washing/cleaning of LC lines, which may lead to instrument downtime and damage with trifluoroacetic acid (TFA) before switching the instrument for the analysis of other samples. All of the above methods follow the same treatment strategy, i.e., an acid extraction protocol involving acetonitrile or methanol or water in different proportions and subjected to reversed-phase SPE (solid-phase extraction) to lower the aggregates of intrusive substances.

Saluti et al. [95] created a novel system for quantification as well as identification of twelve aminoglycosides (AGs) and two colistins in bovine meat and milk through liquid chromatography combined with quadrupole-orbitrap mass spectrometry and hydrophilic interaction liquid chromatography (HILIC). In HILIC, bare silica poroshell 120 showed the optimum result and the recoveries of all the drugs were near $72-87 \%$ in meat (except colistins) and $82-96 \%$ in milk. In another study, an efficient analytical system was created for the simultaneous determination of seven cyclopolypeptide antibiotics (vancomycin, polymyxin B, polymyxin E, teicoplanin A2, cacitracin A, daptomycin, and virginiamycin M1) using liquid chromatography-tandem mass spectrometry [93].

LC-MS/MS and HPLC have enabled researchers to precisely identify colistin from biological entities, but it requires skilled workforce and massive sample pre-treatment, involving both solid-phase extraction and protein precipitation as shown in Figure 3.

These techniques are mainly used for laboratory examination and are not employed for robust screening of bulky samples.

A microbiological technique, i.e., screening test for antibiotic residues (STAR), was developed to analyze the milk samples spiked with eight different concentrations of colistin according to the sensitivity of bacterial strains against this antibiotic. The detection limit of this approach was found to be $1 \mathrm{mg} / \mathrm{L}$. During authentication of the STAR protocol, the reading of colistin in milk was measured to be 200-2000-fold more than its maximum residue limit $\left(50 \mu \mathrm{gL}^{-1}\right)$, thus leading to rejection of this protocol for colistin detection $[98,99]$. 


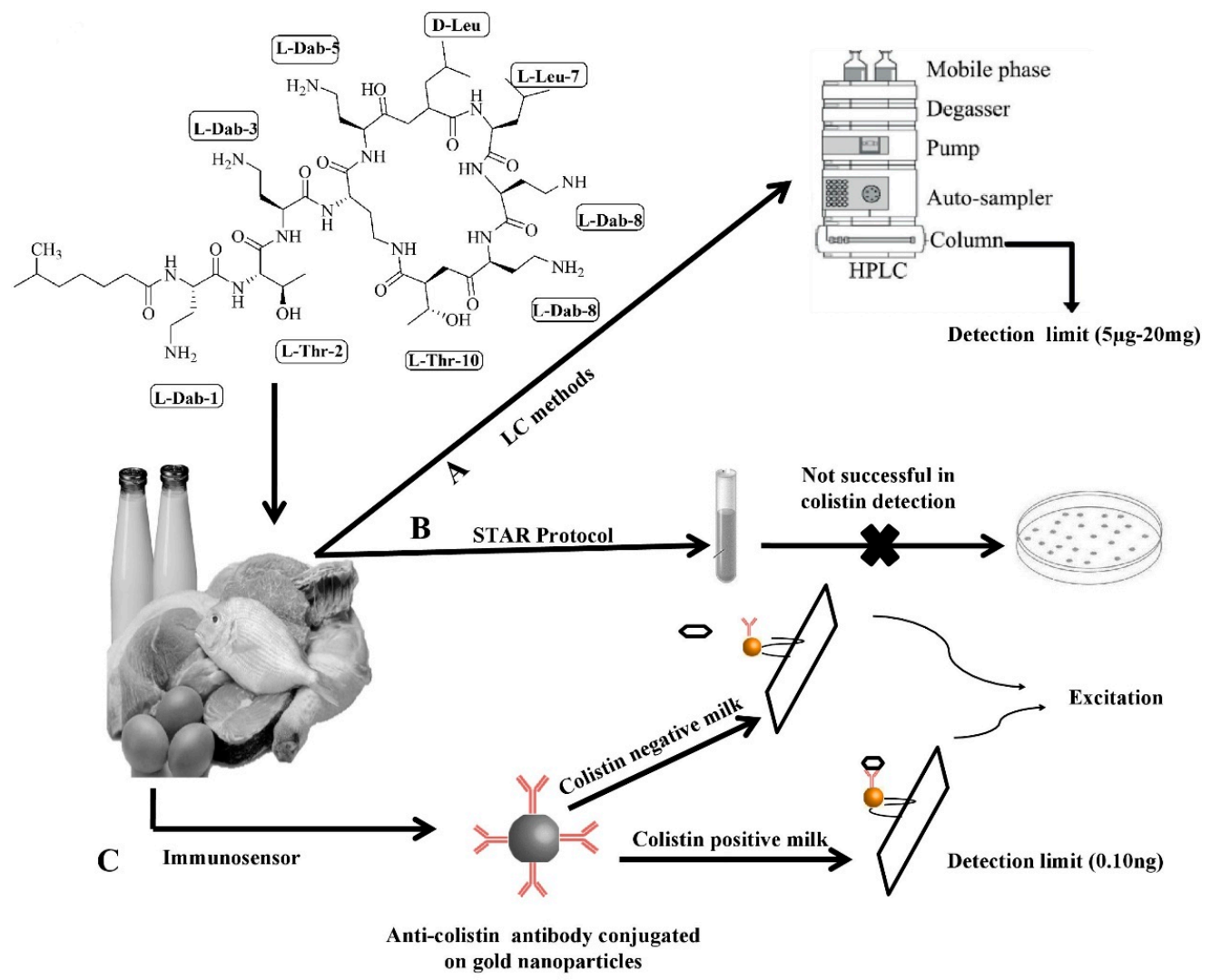

Figure 3. Graphical illustration of different methods used for colistin detection in animal-based food.

\section{Conclusions and Future Outlook}

Colistin has been identified as an imperative alternative for MDR Gram-negative microbes. However, the emergence of colistin-resistant strains has created the havok as it is the last resort for treating infection. Moreover, many reports have linked the colistin resistance with inadequate dosing. The challenges have made us realize the importance of optimized dosage, exclusively in chronically ill patients with MDR strains. The resistant strains of colistin remain the matter of great concern and make it of utmost importance to detect the colistin in food animals. However, there are very few conventional methods available for the detection of colistin use in animal-based food and other livestock. Most of these methods can detect colistin up to a sensitivity limit. The laboratory check-ups to assess the effective use of colistin treatment at farms need to be highlighted by veterinarians. The data on the usage of colistin in animal-based food are of vital necessity, as it supplies a base for the evolution of national policies and also elucidates the hazards of colistin resistance management and evaluates the effect of possible involvement [100,101].

Recently, a surface-enhanced Raman scattering (SERS) immune-sensor was developed for the detection of colistin in milk [15]. In this method, 5,5-dithiobis-2-nitrobenzoic acid (DTNB) was labeled on gold nanoparticles along with anti-colistin monoclonal antibody $(\mathrm{mAb})$. The SERS immune-sensor was attached to the lateral flow strip, which was further attached with Raman signal readout to quantify the colistin amount with high precision. This method can detect concentrations as low as $0.10 \mathrm{ng} / \mathrm{mL}$ colistin in milk, which is higher than the value obtained earlier using ELISA and also the maximum residue limit determined by the European Union. Additionally, the spiking experiments displayed a high accuracy of the SERS immune-sensor, with a recovery of $88.1-112.7 \%$ with a standard deviation of less than $15 \%$. This approach has an advantage in terms of robustness and time of detection (below $20 \mathrm{~min}$ ) over the conventional techniques.

It is wellknown that there is dire need for improvement in colistin detection with high accuracy and specificity in animal food. Few bio-sensors have been developed for identifying food-related 
disorders, like the transglutaminase-based nano-sensor for the prognosis of the celiac disorder, as well as human pathogens, e.g., quick detection of Streptococcus pyogenes and Leptospirainterrogans [102-104]. New improvements and novel changes are required in techniques based on precision and specificity to fulfil the future demand for colistin detection and to develop novel biosensors for rapid identification of colistin in animal-based food.

Supplementary Materials: The following are available online at http://www.mdpi.com/2076-2615/10/10/1892/s1, Table S1. Antibiogram pattern of pathogenic microbes against colisitn isolated from patients.

Author Contributions: Conceptualization, B.-H.C., K.K., and D.K.; Manuscript writing, H.K.; Manuscript editing, E.N., R.N., A.K (Anil Kumar)., D.S.D., N.K.U., and R.V.; Critical revising, B.-H.C., K.K., A.K (Ankur Kaushal)., S.K.B., V.K., and D.K. All authors have read and agreed to the published version of the manuscript.

Funding: This research was funded by the University of Hradec Kralove (Faculty of Science VT2019-2021.

Acknowledgments: We acknowledge the University of Hradec Kralove (Faculty of Science, VT2019-2021) and UHHK, 00179906).

Conflicts of Interest: The authors declare no conflict of interest.

\section{References}

1. Komura, S.; Kurahashi, K. Partial-purification and properties of L-2,4-diaminobutyric acid activating enzyme from a polymyxin-E producing organism. J. Biochem. 1979, 86, 1013-1021. [CrossRef]

2. Falagas, M.E.; Kasiakou, S.K. Colistin: The revival of polymyxins for the management of multidrug-resistant gram-negative bacterial infections. Clin. Infect. Dis. 2005, 40, 1333-1341. [CrossRef]

3. Newton, B.A. Properties and mode of action of the polymyxins. Bacteriol. Rev. 1956, 20, 14-27. [CrossRef]

4. Schindler, M.; Osborn, M.J. Interaction of divalent cations and polymyxin B with lipopolysaccharide. Biochemistry 1979, 18, 4425-4430. [CrossRef]

5. Koch-Weser, J.; Sidel, V.W.; Federman, E.B.; Kanarek, P.; Finer, D.C.; Eaton, A.E. Adverse effects of sodium colistimethate. Manifestations and specific reaction rates during 317 courses of therapy. Ann. Intern. Med. 1970, 72, 857-868. [CrossRef]

6. Spapen, H.; Jacobs, R.; Van-Gorp, V.; Troubleyn, J.; Honoré, P.M. Renal and neurological side effects of colistin in critically ill patients. Ann. IntensiveCare. 2011, 1, 14. [CrossRef]

7. Poirel, L.; Jayol, A.; Nordmann, P. Polymyxins: Antibacterial activity, susceptibility testing, and resistance mechanisms encoded by plasmids or chromosomes. Clin. Microbiol. Rev. 2017, 30, 557-596. [CrossRef]

8. EFSA European Food Safety Authority; ECDC (European Centre for Disease Prevention and Control). The European Union summary report on trends and sources of zoonoses, zoonotic agents and food-borne outbreaks in 2016. EFSA J. 2017, 15, 5077.

9. Aviv, G.; Tsyba, K.; Steck, N.; Salmon-Divon, M.; Cornelius, A.; Rahav, G.; Grassl, A.G.; Gal-Mor, O. A unique megaplasmid contributes to stress tolerance and pathogenicity of an emergent Salmonella enterica serovar Infantis strain. Environ. Microbiol. 2014, 16, 977-994. [CrossRef] [PubMed]

10. Franco, A.; Leekitcharoenphon, P.; Feltrin, F.; Alba, P.; Cordaro, G.; Iurescia, M.; Tolli, R.; D’Incau, M.; Staffolani, M.; Giannatale, E.D.; et al. Emergence of a clonal lineage of multidrug-resistant ESBL-producing Salmonella infantis transmitted from broilers and broiler meat to humans in Italy between 2011 and 2014. PLoS ONE 2015, 10, e0144802. [CrossRef] [PubMed]

11. Hindermann, D.; Gopinath, G.; Chase, H.; Negrete, F.; Althaus, D.; Zurfluh, K.; Tall, B.D.; Stephan, R.; Nüesch-Inderbinen, M. Salmonella enterica serovar infantis from food and human infections, Switzerland, 2010-2015: Poultry-related multidrug resistant clones and an emerging ESBL producing clonal lineage. Front. Microbiol. 2017, 8, 1322. [CrossRef] [PubMed]

12. Tate, H.; Folster, J.P.; Hsu, C.H.; Chen, J.; Hoffmann, M.; Li, C.; Morales, C.; Tyson, G.H.; Mukherjee, S.; Brown, A.C.; et al. Comparative analysis of extended spectrum beta-lactamase CTX-M-65-producing Salmonella infantis isolates from humans, food animals, and retail chickens in the United States. Antimicrob. Agents Chemother. 2017, 61, e00488-e00517. [CrossRef] [PubMed]

13. Lozano-Leon, A.; Garcia-Omil, C.; Dalama, J.; Rodriguez-Souto, R.; Martinez-Urtaza, J.; Gonzalez-Escalona, N. Detection of colistin resistance $m c r-1$ gene in Salmonella enterica serovar Rissen isolated from mussels, Spain, 2012-2016. Eurosurveillance 2019, 24, 1900200. [CrossRef] [PubMed] 
14. Mazutti, K.; Costa, L.B.; Nascimento, L.V.; Filho, T.F.; Beirão, B.C.B.; Júnior, P.C.M.; Maiorka, A. Effect of colistin and tylosin used as feed additives on the performance, diarrhea incidence, and immune response of nursery pigs. Semin. Cienc Agrar. 2016, 37, 1947-1962. [CrossRef]

15. Li, Y.; Tang, S.; Zhang, W.; Cui, X.; Zhang, Y.; Jin, Y.; Zhang, X.; Chen, Y. A surface-enhanced Raman scattering-based lateral flow immunosensor for colistin in raw milk. Sens. Actuat. B Chem. 2019, 282, 703-711. [CrossRef]

16. Laxminarayan, R.; Matsoso, P.; Pant, S.; Brower, C.; Røttingen, J.A.; Klugman, K.; Davies, S. Access to effective antimicrobials: A worldwide challenge. Lancet 2016, 387, 168-175. [CrossRef]

17. Shen, Z.; Wang, Y.; Shen, Y.; Shen, J.; Wu, C. Early emergence of $m c r-1$ in Escherichia coli from food-producing animals. Lancet Infect. Dis. 2016, 16, 293. [CrossRef]

18. Maron, D.F.; Smith, T.J.; Nachman, K.E. Restrictions on antimicrobial use in food animal production: An international regulatory and economic survey. Global Health. 2013, 9, 48. [CrossRef]

19. EMA/AMEG. Updated Advice on the Use of Colistin Products in Animals within the European Union: Development of Resistance and Possible Impact on Human and Animal Health. Available online: https://www.ema.europa.eu/en/documents/scientific-guideline/updated-advice-usecolistin-products-animals-within-european-union-development-resistance-possible_en-0.pdf (accessed on 9 August 2019).

20. ECDC/EFSA/EMA. ECDC/EFSA/EMA Second Joint Report on the Integrated Analysis of the Consumption of Antimicrobial Agents and Occurrence of Antimicrobial Resistance in Bacteria from Humans and Food-Producing Animals. Available online: https://www.ema.europa.eu/en/documents/report/ecdc/ efsa/ema-second-joint-report-integrated-analysis-consumption-antimicrobial-agents-occurrence_en.pdf (accessed on 9 August 2019).

21. Friedlander, L.G.; Arnold, D. Colistin. Available online: http://www.fao.org/fileadmin/user_upload/vetdrug/ docs/2-2006-colistin.pdf (accessed on 13 August 2019).

22. Kempf, I.; Fleury, M.A.; Drider, D.; Bruneau, M.; Sanders, P.; Chauvin, C.; Madec, J.Y.; Jouy, E. What do we know about resistance to colistin in Enterobacteriaceae in avian and pig production in Europe? Int. J. Antimicrob. Agents 2013, 42, 379-383. [CrossRef]

23. Catry, B.; Cavaleri, M.; Baptiste, K.; Grave, K.; Grien, K.; Holm, A.; Jukes, H.; Liebana, E.; Navas, A.L.; Mackay, D.; et al. Use of colistin-containing products within the European union and European economic area(EU/EEA): Development of resistance in animals and possible impact on human and animal health. Int. J. Antimicrob. Agents 2015, 46, 297-306. [CrossRef]

24. Barbieri, N.; Nielsen, D.W.; Wannemuehler, Y.; Cavender, T.; Hussein, A.; Yan, S.G.; Nolan, L.K.; Logue, C.M. mcr-1 identified in avian pathogenic Escherichia coli (APEC). PLoS ONE 2017, 12, e0172997. [CrossRef]

25. Botsoglou, N.A.; Fletouris, D.J. Antibacterial drugs. In Drug Residues in Foods; Fletouris, D., Botsoglou, N., Eds.; CRC Press: London, UK, 2000; pp. 27-103.

26. Goetting, V.; Lee, K.A.; Tell, L.A. Pharmacokinetics of veterinary drugs in laying hens and residues in eggs: A review of the literature. J. Vet. Pharmacol. Ther. 2011, 34, 521-556. [CrossRef]

27. Nolan, L.K.; Barnes, H.J.; Vaillancourt, J.P.; Abdul-Aziz, T.; Louge, C.M. Colibacillosis. In Diseases of Poultry; Swayne, D., Ed.; JohnWiley \& Sons, Ltd.: Chichester, UK, 2007; pp. 751-805.

28. Löhren, U.; Ricci, A.; Cummings, T.S. Guidelines for antimicrobial use in poultry. In Guide to Antimicrobial Use in Animals; Luca, G., Jensen, L.B., Kruse, H., Eds.; BlackwellPublishing, Ltd.: Oxford, UK, 2009; pp. $126-142$.

29. EU. On Pharmacologically Active Substances and Their Classification Regarding Maximum Residue Limits in Food Stuffs of Animal Origin. Available online: https:/ec.europa.eu/health/sites/health/files/files/eudralex/ vol-5/reg_2010_37/reg_2010_37_en.pdf (accessed on 7 August 2019).

30. FSSAI. Food Safety and Standards (Contaminants, ToxinsandResidues). Available online: https://archive. fssai.gov.in/home/fss-legislation/fss-regulations.html (accessed on 7 August 2019).

31. MAC. National Standard of the People's Republic of China. Available online: http://www.agrichina.org/ admin/kindeditor-4.1.2/attached/file/20160617/20160617183721_1681.pdf (accessed on 7 August 2019).

32. PHR. Available online: https://www.elegislation.gov.hk/hk/cap139N (accessed on 7 August 2019).

33. JFCRF. Maximum Residue Limitsin Animalsand Fishery Products. Available online: http://db.ffcr.or.jp/front/ food_group_comp (accessed on 7 August 2019).

34. Ziv, G.; Nouws, F.M.; Van Ginnekin, C.A.M. The pharmacokinetics and tissue levels of polymyxin B, colistin and gentamicin in calves. J. Vet. Pharmacol. Ther. 1982, 5, 45-58. [CrossRef] [PubMed] 
35. Blood, D.C.; Radostits, O.M. Practical antimicrobial therapeutics: Polymyxin B and colistin. In Veterinary Medicine; Tindall, B., Ed.; London, UK, 1989; p. 151. Available online: http://www.fao.org/fileadmin/user_ upload/vetdrug/docs/2-2006-colistin.pdf (accessed on 17 July 2019).

36. Archimbault, P.; Boutier, C.; Fellous, R.; Muscat, G. Etude pharmacocinétique de la colistine chez les bovins. Rec Méd. Vét. 1980, 156, 621-626.

37. Escoula, L.; Coste, M.; Larrieu, G. Biodisponibilité de l'association érythromycinecolistine chez les veaux. Ann. Rech. Vet. 1981, 12, 321-326. [PubMed]

38. Ziv, G.; Sulman, F.G. Passage of polymyxin from serum into milk in Ewes. Am. J. Vet. Res. 1973, 34, 317-322.

39. Al-Khayyat, A.A.; Aronson, A.L. Pharmacologic and toxicologic studies with the polymyxins.II.Comparative pharmacologic studies of the sulfate and methanesulfonate salts of polymyxin B and colistin in dogs. Chemotherapy 1973, 19, 92-97. [CrossRef] [PubMed]

40. Sato, H.; Ouchi, M.; Koumi, J. Studies on the distribution of colistin sulfate in the body. Distribution and change with time in chickens and pigs by oral administration. Jpn. J. Antibiot. 1972, 25, 239-245.

41. Terakado, S.; Azechi, H.; Omae, K.; Koyama, T.; Ninomiya, K.; Kashiwazaki, M. Distribution of colistin sulfate and changes with time in intestinal E.coli counts in pigs following oral administration. Seventy-Third Congr. Jpn. Soc. Vet. Med. 1972, 5-22.

42. Lin, B.; Zhang, C.; Xiao, X. Toxicity, bioavailability and pharmacokinetics of a newly formulated colistin sulfate solution. J. Vet. Pharm. Ther. 2005, 28, 349-354. [CrossRef]

43. Guyonnet, J.; Manco, B.; Baduel, L.; Kaltsatos, V.; Aliabadi, M.H.F.S.; Lees, P. Determination of a dosage regimen of colistin by pharmacokinetic/pharmacodynamic integration and modeling for treatment of G.I.T.disease in pigs. Res. Vet. Sci. 2010, 88, 307-314. [CrossRef] [PubMed]

44. Rhouma, M.; Beaudry, F.; Thériault, W.; Bergeron, N.; Laurent-Lewandowski, S.; Fairbrother, J.M.; Letellier, A. Gastric stability and oral bioavailability of colistin sulfate in pigs challenged or not with Escherichia coli O149:F4(K88). Res. Vet. Sci. 2015, 102, 173-181. [CrossRef] [PubMed]

45. Rhouma, M.; Beaudry, F.; Thériault, W.; Bergeron, N.; Beauchamp, G.; Laurent-Lewandowski, S.; Fairbrother, J.M.; Letellier, A. In vivo therapeutic efficacy and pharmacokinetics of colistin sulfate in an experimental model of enterotoxigenic Escherichia coli infection in weaned pigs. Vet. Res. 2016, 47, 58. [CrossRef]

46. Rhouma, M.; Beaudry, F.; Letellier, A. Resistance to colistin: What is the fate for this antibiotic in pig production? Int. J. Antimicrob. Agents 2016, 48, 119-126. [CrossRef]

47. Olaitan, A.O.; Morand, S.; Rolain, J.M. Mechanisms of polymyxin resistance: Acquired and intrinsic resistance in bacteria. Front. Microbiol. 2014, 5, 643. [CrossRef] [PubMed]

48. Delannoy, S.; Le, D.L.; Jouy, E.; Fach, P.; Drider, D.; Kempf, I. Characterization of colistin-resistant Escherichia coli isolated from diseased pigs in France. Front. Microbiol. 2017, 8, 2278. [CrossRef]

49. Liu, Y.Y.; Wang, Y.; Walsh, T.R.; Yi, L.X.; Zhang, R.; Spencer, J.; Doi, Y.; Tian, G.; Dong, B.; Huang, X.; et al. Emergence of plasmid-mediated colistin resistance mechanism $m c r-1$ in animals and human beings in China: A microbiological and molecular biological study. Lancet Infect. Dis. 2016, 16, 161-168. [CrossRef]

50. Biswas, S.; Brunel, J.M.; Dubus, J.C.; Reynaud-Gaubert, M.; Rolain, J.M. Colistin: An update on the antibiotic of the 21st century. Expert Rev. Anti-Infect Ther. 2012, 10, 917-934. [CrossRef]

51. Xavier, B.B.; Lammens, C.; Ruhal, R.; Kumar-Sing, S.; Butaye, P.; Goossens, H.; Malhotra-Kumar, S. Identification of a novel plasmid-mediated colistin-resistance gene, mor-2, in Escherichia coli, Belgium, June 2016. Eurosurveillance 2016, 21, 30280. [CrossRef]

52. AbuOun, M.; Stubberfield, E.J.; Duggett, N.A.; Kirchner, M.; Dormer, L.; Nunez-Garcia, J.; Randall, L.P.; Lemma, F.; Crook, D.W.; Teale, C.; et al. mcr-1 and mcr-2 variant genes identified in Moraxella species isolated from pigs in Great Britain from 2014 to 2015. J. Antimicrob. Chemother. 2017, 72, 2745-2749. [CrossRef]

53. Borowiak, M.; Fischer, J.; Hammerl, J.A.; Hendriksen, R.S.; Szabo, I.; Malorny, B. Identification of a novel transposon-associated phosphoethanolamine transferase gene, $m c r-5$,conferring colistin resistance in d-tartrate fermenting Salmonella enterica subsp. Enterica serovar Paratyphi B. J. Antimicrob. Chemother. 2017, 72, 3317-3324. [CrossRef]

54. Yin, W.; Li, H.; Shen, Y.; Liu, Z.; Wang, S.; Shen, Z.; Zhang, R.; Walsh, T.R.; Shen, J.; Wang, Y. Novel plasmid-mediated colistin resistance gene mcr-3 in Escherichia coli. MBio 2017, 8. [CrossRef] [PubMed] 
55. Teo, J.W.P.; Kalisvar, M.; Venkatachalam, I.; Ng, O.T.; Lin, R.T.P.; Octavia, S. mcr-3 and mcr-4 variants in carbapenemase-producing clinical Enterobacteriaceae do not confer phenotypic polymyxin resistance. J. Clin. Microbiol. 2018, 56. [CrossRef] [PubMed]

56. Wang, X.; Wang, Y.; Zhou, Y.; Li, J.; Yin, W.; Wang, S.; Zhang, S.; Shen, J.; Wang, Y. Emergence of a novel mobile colistin resistance gene, mor-8, in NDM-producing Klebsiella pneumoniae. Emerg. Microbes Infect. 2018, 7, 122. [CrossRef] [PubMed]

57. Yang, Y.Q.; Li, Y.X.; Lei, C.W.; Zhang, A.Y.; Wang, H.N. Nove lplasmid-mediated colistin resistance gene mcr-7.1 in Klebsiella pneumoniae. J. Antimicrob. Chemother. 2018, 73, 1791-1795. [CrossRef]

58. Joshi, P.R.; Thummeepak, R.; Paudel, S.; Acharya, M.; Pradhan, S.; Banjara, M.R.; Leungtongkam, U.; Sitthisak, S. Molecular characterization of colistin-resistant Escherichia coli isolated from chickens: First report fromNepal. Microb. Drug Resist. 2019, 25, 846-854. [CrossRef]

59. Yassin, A.K.; Zhang, J.; Wang, J.; Chen, L.; Kelly, P.; Butaye, P.; Lu, G.; Gong, J.; Li, M.; Wang, Y.; et al. Identification and characterization of $\mathrm{mcr}$ mediated colistin resistance in extra intestinal Escherichia coli from poultry and live stock in China. FEMS Microbiol. Lett. 2017, 364, fnx242. [CrossRef]

60. Pishnian, Z.; Haeili, M.; Feizi, A. Prevalence and molecular determinants of colistin resistance among commensal Enterobacteriaceae isolated from poultry in northwest of Iran. Gut Pathog. 2019, 11, 2. [CrossRef]

61. Nguyen, N.T.; Nguyen, H.M.; Nguyen, C.V.; Nguyen, T.V.; Nguyenm, M.T.; Thai, H.Q.; Ho, M.H.; Thwaites, G.; Ngo, H.T.; Baker, S.; et al. Use of colistin and other critical antimicrobials on pig and chicken farms in Southern Vietnam and its association with resistance in commensal Escherichia col i bacteria. Appl. Environ. Microbiol. 2016, 82, 3227-3235. [CrossRef]

62. Monte, D.F.; Mem, A.; Fernandes, M.R.; Cerdeira, L.; Esposito, F.; Galvão, J.A.; Franco, B.D.G.M.; Lincopan, N.; Landgraf, M. Chicken meat as a reservoir of colistin-resistant Escherichia coli strains carrying mcr-1 genes in South America. Antimicrob. Agents Chemother. 2017, 61. [CrossRef]

63. Hasman, H.; Hammerum, A.M.; Hansen, F.; Hendriksen, R.S.; Olesen, B.; Agersø, Y.; Zankari, E.; Leekitcharoenphon, P.; Stegger, M.; Kass, R.S.; et al. Detection of mcr-1 encoding plasmid-mediated colistin resistant Escherichiacoli isolates from human bloodstream infection and imported chicken meat, Denmark 2015. Eurosurveillance 2015, 20, 30085. [CrossRef] [PubMed]

64. Quesada, A.; Ugarte-Ruiz, M.; Iglesias, M.R.; Porrero, M.C.; Martínez, R.; Florez-Cuadrado, D.; Campos, M.J.; García, M.; Píriz, S.; Sáez, J.L.; et al. Detection of plasmid mediated colistin resistance(MCR-1) in Escherichia coli and Salmonella enterica isolated from poultry and swine in Spain. Res. Vet. Sci. 2016, 105, 134-135. [CrossRef] [PubMed]

65. Donà, V.; Bernasconi, O.J.; Pires, J.; Collaud, A.; Overesch, G.; Ramette, A.; Perreten, V.; Endimiani, A. Heterogens genetic location of $m c r-1$ in colistin-resistant Escherichia coli isolates from humans and retail chicken meat in Switzerland: Emergence of $m c r$-1-carrying Inc K2 plasmids. Antimicrob. Agents Chemother. 2017, 61. [CrossRef] [PubMed]

66. Schwaiger, K.; Huther, S.; Hölzel, C.; Kämpf, P.; Bauer, J. Prevalence of antibiotic-resistant Enterobacteriaceae isolated from chicken and pork meat purchased at the slaughter house and at retail in Bavaria, Germany. Int. J. Food Microbiol. 2012, 154, 206-211. [CrossRef] [PubMed]

67. Kusumoto, M.; Ogura, Y.; Gotoh, Y.; Iwata, T.; Hayashi, T.; Akiba, M. Colistin-resistant mcr-1-positive pathogenic Escherichia coli in swine, Japan,2007-2014. Emerg. Infect. Dis. 2016, 22, 1315-1317. [CrossRef]

68. Benameura, Q.; Guemourb, D.; Hammoudic, A.; Aoudiad, H.; Aggad, H.; Humblet, M.F.; Saegerman, C. Antimicrobial resistance of Escherichia coli isolated from chickens in West of Algeria. Int. J. Sci. Basic Appl. Res. 2014, 13, 366-370.

69. Chiou, C.S.; Chen, Y.T.; Wang, Y.W.; Liu, Y.Y.; Kuo, H.C.; Tu, Y.H.; Lin, A.C.; Liao, Y.S.; Hong, Y.P. Dissemination of mcr-1-carrying plasmids among colistin-resistant Salmonella strains from humans and food-producing animals in Taiwan. Antimicrob. Agents Chemother. 2017, 61. [CrossRef]

70. Anjum, M.F.; Duggett, N.A.; AbuOun, M.; Randall, L.; Nunez-Garcia, J.; Ellis, R.J.; Rogers, J.; Horton, R.; Brena, C.; Williamson, S.; et al. Colistin resistance in Salmonella and Escherichia coli isolates from a pig farm in Great Britain. J. Antimicrob. Chemother. 2016, 71, 2306-2313. [CrossRef]

71. Enne, V.I.; Cassar, C.; Sprigings, K.; Woodward, M.J.; Bennett, P.M. A high prevalence of antimicrobial resistant Escherichia coli isolated from pigs and a low prevalence of antimicrobial resistant E.coli from cattle and sheep in Great Britain at slaughter. FEMS Microbiol. Lett. 2008, 278, 193-1999. [CrossRef] 
72. Curcio, L.; Luppi, A.; Bonilauri, P.; Gherpelli, Y.; Pezzotti, G.; Pesciaroli, M.; Magistrali, C.F. Detection of the colistin resistance gene $m c r-1$ in pathogenic Escherichia coli from pigs affected by post-weaning diarrhoea in Italy. J. Glob. Antimicrob. Resist. 2017, 10, 80-83. [CrossRef]

73. Magwira, C.A.; Gashe, B.A.; Collison, E.K. Prevalence and antibiotic resistance profiles of Escherichia coli O157:H7 in beef products from retail outlets in Gaborone, Botswana. J. FoodProt. 2005, 68, 403-406. [CrossRef] [PubMed]

74. Waghamare, R.N.; Paturkar, A.M.; Vaidya, V.M.; Zende, R.J.; Dubal, Z.N.; Dwivedi, A.; Gaikwad, R.V. Phenotypic and genotypic drug resistance profile of Salmonella serovars isolated from poultry farm and processing units located in and around Mumbai city, India. Vet. World. 2018, 11, 1682-1688. [CrossRef] [PubMed]

75. Mir, I.A.; Kashyap, S.K.; Maherchandani, S. Isolation, serotype diversity and antibiogram of Salmonella enterica isolated from different species of poultry in India. Asian Pac. J. Trop. Biomed. 2015, 5, 561-567. [CrossRef]

76. Giani, T.; Arena, F.; Vaggelli, G.; Conte, V.; Chiarelli, A.; Angelis, L.H.D.; Fornaini, R.; Grazzini, M.; Niccolini, F.; Pecile, P.; et al. Large nosocomial outbreak of colistin-resistant, carbapenemase-producing Klebsiella pneumoniae traced to clonal expansion of an mgrB deletion mutant. J. Clin. Microbiol. 2015, 53, 3341-3344. [CrossRef]

77. Landman, D.; Georgescu, C.; Martin, D.A.; Quale, J. Polymyxins revisited. Clin. Microbiol. Rev. 2008, 21, 449-465. [CrossRef]

78. Lim, L.M.; Ly, N.; Anderson, D.; Yang, J.C.; Macander, L.; Jarkowski, A.; Forrest, A.; Bulitta, J.B.; Tsuji, B.T. Resurgence of colistin: A review of resistance, toxicity, pharmacodynamics, and dosing. Pharmacotherapy 2010, 30, 1279-1291. [CrossRef]

79. Yahav, D.; Farbman, L.; Leibovici, L.; Paul, M. Colistin: New lessons on an old antibiotic. Clin. Microbiol. Infect. 2012, 18, 18-29. [CrossRef]

80. Davies, M.; Walsh, T.R. A colistin crisis in India. Lancet Infect. Dis. 2018, 18, 256-257. [CrossRef]

81. Livemint. Govt May Ban Antibiotic Colistin Used to Fatten Chicken. Available online: https://www.livemint. com/Industry/yt5eE5hqMLYP1px2d63Q1K/Govt-may-ban-antibiotic-colistin-used-to-fatten-chicken.html (accessed on 20 June 2019).

82. TOI. Tolerance Limits' to Be Fixed by Food Regulator for Presence of Antibiotics in Animal, Foods. Available online: https://www.fssai.gov.in/upload/media/FSSAI_News_AntiBiotics_TOI_01_08_2018.pdf (accessed on 12 June 2019).

83. FSSAI. Direction under Section 16(5)Read with 18(2)(d)of Food Safety and Standard Act, 2006 Regarding Operationalisation of Draft Food Safety and Standard (Contaminants, Toxins and Residues) Amendment Regulations. 2019. Available online: https://www.fssai.gov.in/upload/advisories/2019/08/ 5d4c042779d77Direction_Colistin_Ban_FSSAI_08_08_2019.pdf (accessed on 8 September 2019).

84. Kunin, C.M. Binding of antibiotics to tissue homogenates. J. Infect. Dis. 1970, 121, 55-64. [CrossRef]

85. Sin, D.W.; Ho, C.; Wong, Y.C.; Ho, S.K.; Ip, A.C.B. Analysis of major components of residual bacitracin and colistin in food samples byliquid chromatography tandem mass spectrometry. Anal. Chim. Acta. 2005, 535, 23-31. [CrossRef]

86. Xu, I.; Tian, X.; Ren, C.; Huang, H.; Zhang, X.; Gong, X.; Liu, H.; Yu, Z.; Zhang, L. Analysis of colistin A and $B$ in fishery products by ultra performance liquid chromatography with positive electro spray ionization tandem mass spectrometry. J. Chromatogr. B 2012, 899, 14-20. [CrossRef] [PubMed]

87. Morovján, G.; CsoKán, P.P.; Németh-Konda, L. HPLC determination of colistin and aminoglycoside antibiotics in feeds by post-column derivatization and fluorescence detection. Chromatographia 1998, 48, 32-36. [CrossRef]

88. Cancho-Grande, B.; Rodríguez-Comesaña, M.; Simal-Gándara, J. Simple HPLC determination of colistin in medicated feeds by pre-column derivatization and fluorescence detection. Chromatographia 2001, 54, 481-484. [CrossRef]

89. Decolin, D.; Leroy, P.; Nicolas, A.; Archimbault, P. Hyphenated liquid chromatographic method for the determination of colistin residues in bovine tissues. J. Chromatogr. Sci. 1997, 35, 557-564. [CrossRef] [PubMed]

90. Fu, Q.; Li, X.; Zheng, K.; Ke, Y.; Wang, Y.; Wang, L.; Yu, F.; Xia, X. Determination of colistin in animal tissues, egg, milk, and feed by ultra-highperformance liquid chromatography-tandem mass spectrometry. Food Chem. 2018, 248, 166-172. [CrossRef] 
91. Meersche, T.V.D.; Pamel, E.V.; Poucke, C.V.; Herman, L.; Heyndrickx, M.; Rasschaert, G.; Daeseleire, E. Development, validation and application of an ultra high performance liquid chromatographic-tandem mass spectrometric method for the simultaneous detection and quantification of five different classes of veterinary antibiotics in swine manure. J. Chromatogr. A. 2016, 1429, 248-257. [CrossRef]

92. Tao, Y.; Xie, S.; Zhu, Y.; Chen, D.; Pan, Y.; Wang, X.; Liu, Z.; Huang, L.; Peng, D.; Yuan, Z. Analysis of major components of bacitracin, colistin and virginiamycin in feed using matrix solid-phase dispersion extraction by liquid chromatography-electrospray ionization tandem mass spectrometry. J. Chromatogr. Sci. 2017, 56, 281-291. [CrossRef]

93. Song, X.; Huang, Q.; Zhang, Y.; Zhang, M.; Xie, J.; He, L. Rapid multi residue analysis of authorized/banned cyclo poly peptide antibiotics in feed by liquid chromatography-tandem mass spectrometry based on dispersives olid-phase extraction. J. Pharm. Biomed. Anal. 2019, 170, 234-242. [CrossRef]

94. Boison, J.O.; Lee, S.; Matus, J. A multi-residue method for the determination of seven polypeptide drug residues in chicken muscle tissues byLC-MS/MS. Anal. Bioanal. Chem. 2015, 406, 4065-4078. [CrossRef]

95. Saluti, G.; Diamanti, I.; Giusepponi, D.; Pucciarini, L.; Rossi, R.; Moretti, S.; Sardella, R.; Galarini, R. Simultaneous determination of aminoglycosides and colistins in food. Food Chem. 2018, 266, 9-16. [CrossRef]

96. Wan, E.C.; Ho, C.; Sin, D.W.; Wong, Y.C. Detection of residual bacitracin A, colistin A, and colist in B in milk and animal tissues by liquid chromatography tandem mass spectrometry. Anal. Bioanal. Chem. 2006, 385, 181-188. [CrossRef]

97. Kaufmann, A.; Widmer, M. Quantitative analysis of polypeptide antibiotic residues in a variety of food matrices by liquid chromatography coupled to tandem mass spectrometry. Anal. Chim. Acta 2013, 797, 81-88. [CrossRef] [PubMed]

98. Gaudin, V.; Maris, P.; Fuselier, R.; Ribouchon, J.L.; Cadieu, N.; Rault, A. Validation of a microbiological method: The STAR protocol, a five-plate test, for the screening of antibiotic residues in milk. Food Addit. Contam. 2004, 21, 422-433. [CrossRef] [PubMed]

99. Gaudin, V.; Hedou, C.; Rault, A.; Verdon, E. Validation of a five plate test, the STAR protocol, for the screening of antibiotic residues in muscle from different animal species according to European decision 2002/657/EC. Food Addit. Contam. 2010, 27, 935-952. [CrossRef] [PubMed]

100. Aarestrup, F.M.; Wegener, H.C.; Collignon, P. Resistance in bacteria of the foodc hain: Epidemiology and control strategies. Expert Rev. Anti-Infect Ther. 2008, 6, 733-750. [CrossRef]

101. Rhouma, M.; Beaudry, F.; Thériault, W.; Letellier, A. Colistin in pig production: Chemistry, mechanism of antibacterial action, microbial resistance emergence, and one health perspectives. Front. Microbiol. 2016, 7, 1789. [CrossRef]

102. Gupta, S.; Kaushal, A.; Kumar, A.; Kumar, D. Ultrasensitive transglutaminase based nanosensor for early detection of celiac disease in human. Int. J. Biol. Macromol. 2017, 5, 905-911. [CrossRef]

103. Kaushal, A.; Singh, S.; Kumar, A.; Kumar, D. Nano-Au/cMWCNT modified speB gene specific amperometric sensor for rapidly detecting Streptococcus pyogenes causing rheumatic heart disease. Indian J. Microbiol. 2017, 57, 121-124. [CrossRef]

104. Nagraik, R.; Kaushal, A.; Gupta, S.; Dhar, P.; Sethi, S.; Kumar, D. Optimized DNA-based bioassay for Leptospira interrogans detection: A novel platform for leptospirosis diagnosis. 3 Biotech 2019, 9, 284. [CrossRef]

Publisher's Note: MDPI stays neutral with regard to jurisdictional claims in published maps and institutional affiliations.

(C) 2020 by the authors. Licensee MDPI, Basel, Switzerland. This article is an open access article distributed under the terms and conditions of the Creative Commons Attribution (CC BY) license (http://creativecommons.org/licenses/by/4.0/). 